\title{
Clinical significance of primary tumor score determined by tumor depth and size in patients with resectable gastric cancer
}

\author{
Naoto Haraguchi ${ }^{1, *}$, Takaaki Arigami ${ }^{2, *}$, Yoshikazu Uenosono $^{1}$, Shigehiro Yanagita ${ }^{1}$, \\ Yasuto Uchikado", Shinichiro Mori ${ }^{1}$, Hiroshi Kurahara ${ }^{1}$, Yuko Kijima ${ }^{1}$, Akihiro \\ Nakajo ${ }^{1}$, Kosei Maemura ${ }^{1}$, Sumiya Ishigami ${ }^{1}$ and Shoji Natsugoe ${ }^{1,2}$ \\ ${ }^{1}$ Department of Digestive Surgery, Breast and Thyroid Surgery, Kagoshima University Graduate School of Medical and Dental \\ Sciences, Kagoshima, Japan \\ ${ }^{2}$ Onco-biological Surgery, Kagoshima University Graduate School of Medical and Dental Sciences, Kagoshima, Japan \\ *These authors contributed equally to this work
}

Correspondence to: Takaaki Arigami, email: arigami@m.kufm.kagoshima-u.ac.jp

Keywords: primary tumor score; tumor size; depth of tumor invasion; prognostic factor; gastric cancer

Received: September 11, 2017 Accepted: November 16, $2017 \quad$ Published: January 04, 2018

Copyright: Haraguchi et al. This is an open-access article distributed under the terms of the Creative Commons Attribution License 3.0 (CC BY 3.0), which permits unrestricted use, distribution, and reproduction in any medium, provided the original author and source are credited.

\section{ABSTRACT}

Although postoperative management of gastric cancer is determined by pathological stage based on the tumor-node-metastasis classification, predicting disease recurrence and prognosis in patients undergoing gastrectomy is clinically difficult. We investigated the depth of tumor invasion and tumor size in resected specimens from patients with gastric cancer and assessed the clinical utility of primary tumor score (PTS) calculated by tumor depth and size as a prognostic marker. We classified 247 patients with gastric cancer into three groups based on cut-off values for deeper tumor invasion (pT2-T4) and larger tumor size ( $\geq 45 \mathrm{~mm}$ ) as a PTS of 2 (both abnormalities), 1 (one abnormality), or 0 (neither abnormality). PTS correlated significantly with lymph node metastasis, lymphovascular invasion, and stage $(P<$ 0.0001 each). Survival differences among groups based on PTS were significant ( $P$ $<0.0001)$. Multivariate analysis identified PTS alone as an independent prognostic factor $(P=\mathbf{0 . 0 3 6 3})$. PTS derived from primary tumor information alone is a potentially useful marker for predicting tumor progression and prognosis in postoperative patients with gastric cancer.

\section{INTRODUCTION}

Gastric cancer is a common gastrointestinal malignancy and the third leading cause of cancer death globally [1]. Although the prognosis of patients with resectable gastric cancer has improved due to the remarkable progress in surgical techniques and chemotherapies, some patients die of recurrent disease after surgery. The need for administration of adjuvant chemotherapy is currently judged based on pathological stage in patients with resectable gastric cancer [2]. According to the criteria of the Japanese Gastric Cancer Treatment Guidelines 2014 (ver. 4), induction of adjuvant chemotherapy is recommended for patients with stage II or III pathology [2]. This therapeutic strategy indicates a high incidence of disease recurrence in postoperative patients with stage II-III. On the other hand, carcinoembryonic antigen (CEA) and carbohydrate antigen 19-9 (CA199) are representative blood markers for the clinical management of patients with gastric cancer. However, the sensitivity and specificity of these conventional tumor markers are clinically insufficient for predicting disease recurrence and prognosis [3, 4]. Consequently, these key issues suggest few prognostic indicators are available for accurately predicting survival outcomes in patients with gastric cancer.

In postoperative management, patients are pathologically categorized and staged based on the criteria of the tumor-node-metastasis (TNM) classification of gastric carcinoma established by the Union for International Cancer Control (UICC) [5]. Pathological TNM stage involving tumor progression and prognosis is comprehensively decided based on the depth of tumor invasion and the presence of lymph node 
metastasis and distant metastasis [5]. Accordingly, we need to grasp three different clinicopathological statuses in order to determine pathological stage based on this classification system.

Several investigators have demonstrated the clinical impact of tumor size as a simple prognostic indicator in patients with resectable gastric cancer [615]. Furthermore, according to a large-scale retrospective analysis of 2405 patients with gastric cancer, tumor size could improve the accuracy of TNM staging in predicting survival [15]. However, few reports have revealed that tumor depth and size have been simultaneously assessed as a predictive marker for tumor progression and prognosis in patients with resectable gastric cancer [16]. If a primary tumor score (PTS) based on both tumor depth and size correlated with TNM stage, we may be able to predict survival outcomes using primary tumor status alone, even without knowing disease status in terms of lymph node metastasis or distant metastasis.

The present study examined tumor depth and size in patients with resectable gastric cancer, and evaluated the relationship between these primary tumor factors and clinicopathological factors. We also assessed the potential utility of PTS based on tumor depth and size as a prognostic indicator for gastric cancer.

\section{RESULTS}

\section{Tumor depth and size as predictors of tumor progression}

We initially examined the relationship between clinicopathological factors and depth of tumor invasion or tumor size to assess their clinical impact during tumor progression in 247 patients with resectable gastric cancer.

Pathological examination demonstrated pT1 in 138 patients, pT2 in 17 patients, pT3 in 55 patients, and pT4 tumors in 37 patients. Table 1 shows the relationship between status of tumor depth and clinicopathological features. Tumor depth correlated significantly with lymph node metastasis, lymphatic and venous invasions, and stage $(P<0.0001$ each $)$.

Tumor size ranged between 1 and $250 \mathrm{~mm}$ in all patients with gastric cancer. Mean tumor size \pm standard deviation (SD) was $49.4 \pm 37.3 \mathrm{~mm}$. Mean tumor size $( \pm \mathrm{SD})$ in $138,17,55$, and 37 patients with pT1, pT2, pT3, and pT4 tumors were $31.3 \pm 22.7,47.1 \pm 33.1$, $64.6 \pm 30.1$, and $95.4 \pm 43.9 \mathrm{~mm}$, respectively (Figure 1A). Tumor size correlated significantly with depth of tumor invasion $(P<0.0001)$. Mean tumor size $( \pm$ SD) was $35.3 \pm 25.1 \mathrm{~mm}$ in 143 patients with lymph node status N0 and $68.8 \pm 42.4 \mathrm{~mm}$ in 104 patients with $\geq$ N1 (Figure 1B). Tumor size was significantly greater in patients with lymph node metastasis than in those without lymph node metastasis $(P<0.0001)$. In addition, tumor size correlated with presence or absence of lymphatic and venous invasions ( $P<0.0001$ each) (Figure $1 \mathrm{C}$ and 1D). Mean tumor size ( \pm SD) was $31.5 \pm 23.2 \mathrm{~mm}$ in 139 patients with stage I, $51.5 \pm 23.0 \mathrm{~mm}$ in 44 patients with stage II, and $86.8 \pm 41.9 \mathrm{~mm}$ in 64 patients with stage III (Figure 1E). Tumor size differed significantly between categorical stages $(P<0.0001)$.

Next, we investigated the potential utility of tumor size for predicting the presence or absence of lymph node metastasis using receiver operating characteristic (ROC) analysis. The area under the curve (AUC) value for discriminating patients with nodal metastasis from those without nodal metastasis based on tumor size was 0.762 , setting a cut-off for tumor size of $45 \mathrm{~mm}$ (Figure $1 F)$. The sensitivity and specificity of tumor size for predicting lymph node metastasis were 0.664 and 0.734 , respectively. We therefore classified all patients into two groups according to tumor size $\geq 45 \mathrm{~mm}(n=107)$; and < $45 \mathrm{~mm}(n=140)$. This binary classification for tumor size was used in the following analyses.

\section{PTS grading system}

A grading system of PTS was defined by both tumor depth and size. According to this grading system, all patients were categorized into three groups based on the depth status of tumor invasion and the cut-off value for tumor size, as follows: PTS 2, both deeper tumor invasion (pT2-T4) and greater tumor size $\geq 45 \mathrm{~mm}$ ); PTS 1 , either deeper tumor invasion or greater tumor size; and PTS 0, neither deeper tumor invasion nor greater tumor size.

According to this PTS grading system, 109 patients (44.1\%) showed PTS 0, 60 patients (24.3\%) showed PTS 1 , and 78 patients $(31.6 \%)$ showed PTS 2.

\section{PTS as a predictor of tumor progression}

PTS correlated significantly with lymph node metastasis, lymphatic invasion, vascular invasion, and stage $(P<0.0001$ each; Table 2$)$.

\section{PTS as a predictor of prognosis}

Five-year survival rates were $95.6 \%$ for PTS 0, 83.3\% for PTS 1, and 70.2\% for PTS 2 (Figure 2). Significant differences in survival were seen according to PTS status $(P<0.0001)$.

\section{Uni- and multivariate analyses for survival}

Tumor size, depth of tumor invasion, lymph node metastasis, stage, PTS, and serum concentrations of CEA and CA19-9 all correlated significantly with prognosis in univariate analysis $(P=0.0032, P<0.0001, P=0.0003$, $P<0.0001, P<0.0001, P=0.0338$, and $P=0.0043$, respectively) (Table 3). Multivariate analysis demonstrated 
Table 1: Relationship between depth of tumor invasion and clinicopathological factors

\begin{tabular}{|c|c|c|c|c|c|}
\hline \multirow{2}{*}{ Factor } & \multicolumn{4}{|c|}{ Depth of tumor invasion } & \multirow{2}{*}{$P$-value } \\
\hline & pT1 $(n=138)$ & pT2 $(n=17)$ & pT3 $(n=55)$ & pT4 $(n=37)$ & \\
\hline \multicolumn{6}{|l|}{ Sex } \\
\hline Male & $90(65.2)$ & $14(82.3)$ & $37(67.3)$ & $23(62.2)$ & 0.5045 \\
\hline Female & $48(34.8)$ & $3(17.7)$ & $18(32.7)$ & $14(37.8)$ & \\
\hline \multicolumn{6}{|l|}{ Age (y) } \\
\hline$\leq 70$ & $72(52.2)$ & $11(64.7)$ & $29(52.7)$ & $25(67.6)$ & 0.3139 \\
\hline$>70$ & $66(47.8)$ & $6(35.3)$ & $26(47.3)$ & $12(32.4)$ & \\
\hline \multicolumn{6}{|c|}{ Lymph node metastasis } \\
\hline Negative & $114(82.6)$ & $8(47.1)$ & $14(25.4)$ & $7(18.9)$ & $<0.0001$ \\
\hline Positive & $24(17.4)$ & $9(52.9)$ & $41(74.6)$ & $30(81.1)$ & \\
\hline \multicolumn{6}{|l|}{ Stage } \\
\hline I & $130(94.2)$ & $9(52.9)$ & $0(0.0)$ & $0(0.0)$ & $<0.0001$ \\
\hline II-III & $8(5.8)$ & $8(47.1)$ & $55(100.0)$ & $37(0.0)$ & \\
\hline \multicolumn{6}{|c|}{ Lymphatic invasion } \\
\hline Negative & $112(81.2)$ & $6(35.3)$ & $10(18.2)$ & $3(8.1)$ & $<0.0001$ \\
\hline Positive & $26(18.8)$ & $11(64.7)$ & $45(81.8)$ & $34(91.9)$ & \\
\hline \multicolumn{6}{|c|}{ Venous invasion } \\
\hline Negative & $125(90.6)$ & $5(29.4)$ & $14(25.4)$ & $3(8.1)$ & $<0.0001$ \\
\hline Positive & $13(9.4)$ & $12(70.6)$ & $41(74.6)$ & 34 (91.9) & \\
\hline
\end{tabular}

pT1: invasion of lamina propria or submucosa; pT2: invasion of muscularis propria; $p$ T3: invasion of subserosa; $\mathrm{pT} 4$ : penetration of serosa or invasion of adjacent structures.

PTS alone as an independent prognostic factor $(P=$ 0.0363; Table 4).

\section{DISCUSSION}

Initially, we assessed the clinical utility of tumor depth and size for predicting tumor progression in patients with resectable gastric cancer. We also proposed PTS as a new prognostic indicator determined solely from tumor depth and size. Finally, we evaluated the clinical significance of PTS as a predictor of tumor progression and prognosis in patients with stage I-III gastric cancer.

Depth of tumor invasion is a well-known prognostic factor in patients with gastric cancer. Unsurprisingly, this study demonstrated close relationships between tumor depth and several clinicopathological factors, such as lymph node metastasis and stage. Moreover, deeper tumor invasion was significantly associated with poor prognosis in the present study (data not shown). These results indicate the prognostic impact of tumor depth derived from primary tumor information in patients with gastric cancer.

We focused on tumor size as an additional prognostic factor in this study. Many investigators have demonstrated that greater tumor size correlates closely with increasing number of positive lymph nodes [6-8,
$10,12,13,15]$. In the present study, tumor size correlated significantly with lymph node status. Interestingly, tumor size showed high sensitivity and specificity for discriminating between the presence and absence of lymph node metastasis. These findings suggest tumor size as a promising marker for predicting nodal status. In addition, it is important to estimate lymph node status by tumor size derived from primary tumor information alone for the clinical management of patients with resectable gastric cancer. Furthermore, a study of 2405 patients with gastric cancer by Zhao et al. reported that tumor size classified into five groups provided an independent prognostic factor according to multivariate analysis [15]. In that study, the 5 -year survival rate was significantly lower in patients with tumor size $\geq 45 \mathrm{~mm}$ than in those with tumor size $<45 \mathrm{~mm}(P=0.003$; data not shown) [15]. Those results indicate that assessment of tumor size may be useful for clinical prediction of tumor aggressiveness, including prognosis.

The most noteworthy point of the present study was that we defined PTS calculated by tumor invasion and size derived from primary tumor information alone. At least metastatic status of lymph nodes and distant organs is needed to determine TNM stage. Ohashi et al. also reported the clinical potential of tumor index (TI) as a marker combining tumor depth and size in patients 
Table 2: Relationship between primary tumor score and clinicopathological factors

\begin{tabular}{|c|c|c|c|c|}
\hline \multirow{2}{*}{ Factor } & \multicolumn{3}{|c|}{ Primary tumor score } & \multirow{2}{*}{$P$-value } \\
\hline & $0(n=109)$ & $1(n=60)$ & $2(n=78)$ & \\
\hline \multicolumn{5}{|l|}{ Sex } \\
\hline Male & $74(67.9)$ & $38(63.3)$ & $52(66.7)$ & 0.8337 \\
\hline Female & $35(32.1)$ & $22(36.7)$ & $26(33.3)$ & \\
\hline \multicolumn{5}{|l|}{ Age (y) } \\
\hline$\leq 70$ & $60(55.1)$ & $31(51.7)$ & $46(59.0)$ & 0.6883 \\
\hline$>70$ & $49(44.9)$ & $29(48.3)$ & $32(41.0)$ & \\
\hline \multicolumn{5}{|c|}{ Depth of tumor invasion } \\
\hline pT1 & $109(100.0)$ & $29(48.4)$ & $0(0.0)$ & $<0.0001$ \\
\hline pT2 & $0(0.0)$ & $11(18.3)$ & $6(7.7)$ & \\
\hline pT3 & $0(0.0)$ & $17(28.3)$ & $38(48.7)$ & \\
\hline pT4 & $0(0.0)$ & $3(5.0)$ & $34(43.6)$ & \\
\hline \multicolumn{5}{|c|}{ Lymph node metastasis } \\
\hline Negative & $92(84.4)$ & $35(58.3)$ & $16(20.5)$ & $<0.0001$ \\
\hline Positive & $17(15.6)$ & $25(41.7)$ & $62(79.5)$ & \\
\hline \multicolumn{5}{|l|}{ Stage } \\
\hline I & $105(96.3)$ & $31(51.7)$ & $3(3.8)$ & $<0.0001$ \\
\hline II-III & $4(3.7)$ & $29(48.3)$ & $75(96.2)$ & \\
\hline \multicolumn{5}{|c|}{ Lymphatic invasion } \\
\hline Negative & $91(83.5)$ & $29(48.3)$ & $11(14.1)$ & $<0.0001$ \\
\hline Positive & $18(16.5)$ & $31(51.7)$ & $67(85.9)$ & \\
\hline \multicolumn{5}{|c|}{ Venous invasion } \\
\hline Negative & $98(89.9)$ & $34(56.7)$ & $15(19.2)$ & $<0.0001$ \\
\hline Positive & $11(10.1)$ & $26(43.3)$ & $63(80.8)$ & \\
\hline
\end{tabular}

pT1: invasion of lamina propria or submucosa; pT2: invasion of muscularis propria; $p$ T3: invasion of subserosa; $p$ T4: penetration of serosa or invasion of adjacent structures.

with gastric cancer [16]. They defined TI as pT category (pT1-4) multiplied by tumor size in millimeters [16]. That study identified a close association between TI and prominent clinicopathological prognostic factors, such as lymphovascular invasion, lymph node metastasis, and disease recurrence [16]. Moreover, they set a cut-off TI of 180 to determine the prognostic impact in two groups of patients [16]. In contrast, we categorized patients into three groups using our PTS grading system. The greatest advantage of the PTS grading system is the ability to strictly stratify patients into three categories of low, intermediate, and high malignant potential. Higher PTS correlated significantly with malignant tumor behaviors, such as the presence of lymph node metastasis and advanced stage. These findings suggest that the PTS grading system has potential as a tool for indicating pathological tumor progression.

Furthermore, the present study assessed the prognostic impact of the PTS grading system. Log-rank testing indicated that prognosis differed significantly among the three groups of PTS 0,1 and $2(P<0.0001)$. Tumor size, depth of tumor invasion, and TNM stage were excluded from multivariate analysis due to confounding factors which might cancel out the efficacy of PTS. Then, only PTS was identified as an independent prognostic factor in multivariate analysis $(P=0.0363)$. Similarly, according to the study for TI, patients with TI $\geq 180$ displayed significantly poorer prognosis than those with TI $<180(P<0.0001)[16]$. These PTS and TI studies suggest that a score combining tumor depth and size determined from the primary tumor alone is useful to predict disease recurrence and prognosis in patients with gastric cancer. If PTS has potential for discriminating subclinical patients with high risk of disease recurrence, this grading system may assist in selecting patients for adjuvant chemotherapy in the postoperative management of gastric cancer.

The present study had several limitations. This preliminary study was based on a retrospective analysis 
Table 3: Univariate analysis for survival

\begin{tabular}{|c|c|c|c|}
\hline Independent factor & Hazard ratio & $95 \% \mathrm{CI}$ & P-value \\
\hline \multicolumn{4}{|l|}{ Age (y) } \\
\hline$\leq 70 />70$ & 1.65 & $0.73-3.72$ & 0.2217 \\
\hline \multicolumn{4}{|l|}{ Tumor size } \\
\hline$<45 \mathrm{~mm} / \geq 45 \mathrm{~mm}$ & 3.48 & $1.50-9.01$ & 0.0032 \\
\hline \multicolumn{4}{|l|}{ Depth of tumor invasion } \\
\hline pT1/pT2-T4 & 12.88 & $3.80-80.40$ & $<0.0001$ \\
\hline \multicolumn{4}{|l|}{ Lymph node metastasis } \\
\hline Negative/Positive & 4.96 & $1.99-14.95$ & 0.0003 \\
\hline \multicolumn{4}{|l|}{ Stage } \\
\hline I/II-III & 8.35 & $2.88-35.36$ & $<0.0001$ \\
\hline \multicolumn{4}{|l|}{ Primary tumor score } \\
\hline $0-1 / 2$ & 5.50 & $2.37-14.22$ & $<0.0001$ \\
\hline \multicolumn{4}{|c|}{ Serum CEA levels $(<5$ ng/mL) } \\
\hline Negative/Positive & 2.59 & $1.08-5.92$ & 0.0338 \\
\hline \multicolumn{4}{|c|}{ Serum CA 19-9 levels $(<37 \mathrm{U} / \mathrm{mL})$} \\
\hline Negative/Positive & 3.96 & $1.59-9.12$ & 0.0043 \\
\hline
\end{tabular}

CI: confidence interval.

Table 4: Multivariate analysis for survival

\begin{tabular}{lccc}
\hline Independent factor & Hazard ratio & $\mathbf{9 5 \%}$ CI & P-value \\
\hline $\begin{array}{l}\text { Lymph node metastasis } \\
\quad \text { Negative/Positive } \\
\begin{array}{l}\text { Primary tumor score } \\
\quad 0-1 / 2\end{array}\end{array}$ & 2.28 & $0.78-7.72$ & 0.1345 \\
$\begin{array}{l}\text { Serum CEA levels }(<5 \mathrm{ng} / \mathrm{mL}) \\
\quad \text { Negative/Positive }\end{array}$ & 2.86 & $1.07-8.53$ & 0.0363 \\
$\begin{array}{l}\text { Serum CA 19-9 levels }(<37 \mathrm{U} / \mathrm{mL}) \\
\quad \text { Negative/Positive }\end{array}$ & 1.95 & $0.74-4.90$ & 0.1701 \\
\hline
\end{tabular}

CI: confidence interval.

of a small population $(n=247)$ in a single institution. Moreover, the median duration of follow-up was only 38 months. These limitations may have resulted in bias, which might have impacted several results in the study. Accordingly, larger validation studies with longer followup periods are required to strengthen our findings. We are presently planning further exploration of the PTS grading system in patients with other gastrointestinal tract cancers.

In conclusion, we proposed a promising prognostic score calculated from tumor depth and size, and demonstrated that PTS correlated closely with both tumor progression and prognosis in patients with gastric cancer. Stratification based on a PTS grading system might contribute to clinical planning of therapeutic strategies to optimize prognosis in patients with gastric cancer.

\section{MATERIALS AND METHODS}

\section{Patients}

Between December 1999 and January 2012, a total of 362 consecutive patients with gastric cancer underwent gastrectomy with lymphadenectomy at Kagoshima University Hospital (Kagoshima, Japan). Inclusion criteria for this study were as follows: 1) gastric adenocarcinoma confirmed by histopathology; 2) patients without endoscopic treatment; 3) patients without palliative resection; 4) patients without preoperative chemo- or radiotherapy; 5) patients without multiple gastric lesions; 6) patients without distant metastasis; and 7) patients without synchronous or metachronous cancer in other 
organs. After applying these criteria, 247 patients (164 men, 83 women; age range, 35-89 years; mean, 67 years) were enrolled in the present study. In surgical procedures,
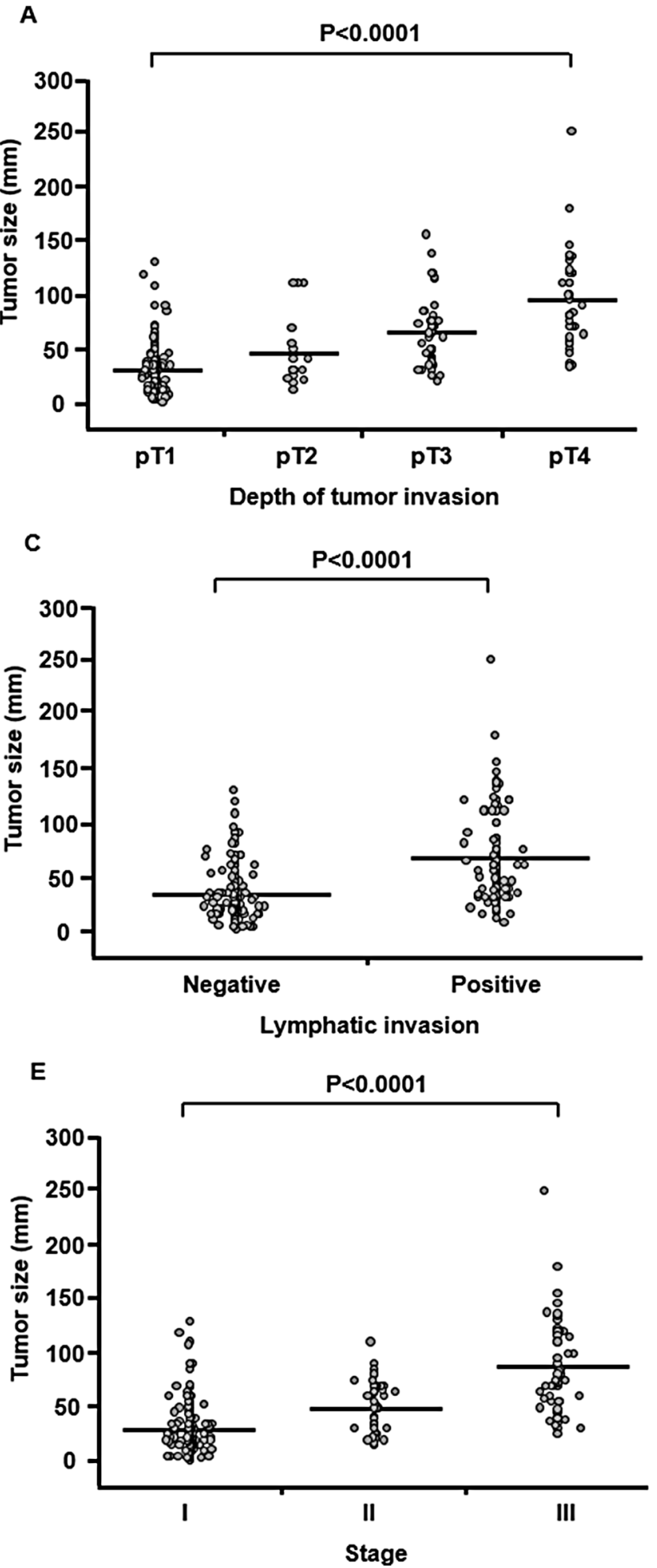

$150(60.7 \%), 24(9.7 \%)$, and $73(29.6 \%)$ patients underwent distal gastrectomy, proximal gastrectomy, and total gastrectomy, respectively. Moreover, the mean
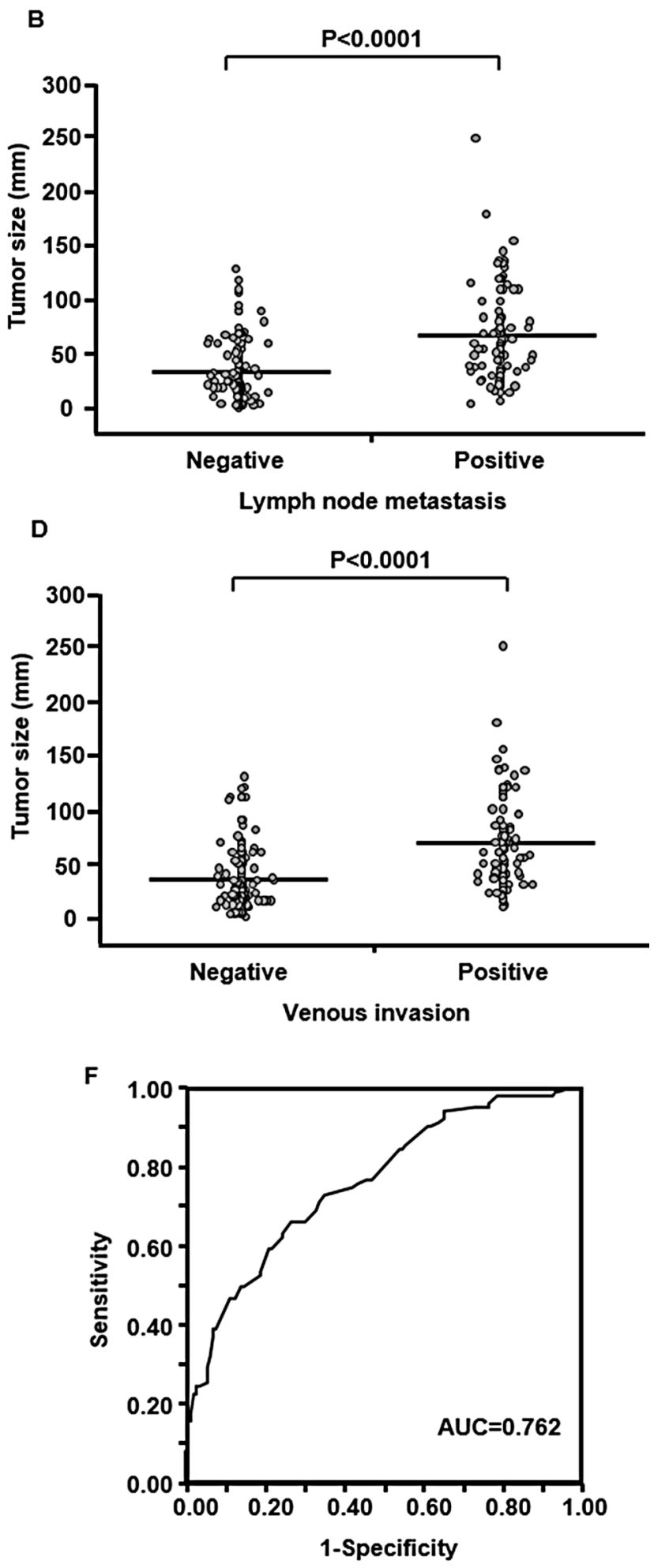

Figure 1: Relationship between tumor size and clinicopathological factors in 247 patients with resectable gastric cancer. Tumor size correlated significantly with depth of tumor invasion (A), lymph node metastasis (B), lymphatic invasion (C), venous invasion (D) and stage (E). Horizontal bars indicate mean tumor size. The receiver operating characteristic curve for discriminating patients with nodal metastasis from patients without nodal metastasis based on tumor size (F). 
number of dissected lymph nodes was 31.5. Patients were classified and staged based on the TNM classification of gastric carcinoma established by the UICC [5]. Table 5 shows the clinicopathological background of participating patients. All patients were followed-up every 3-6 months after surgery by regular clinical examinations at Kagoshima University Hospital, including tumor marker studies (CEA and CA19-9), radiography, ultrasonography, and computed tomography. The median duration of follow-up was 38 months (range, 1-123 months).

This retrospective observational study was approved by the Ethics Committee of Kagoshima University and all patients provided written informed consent to the use of their information.

\section{Assessment of tumor depth and size}

Depth of tumor invasion was assessed based on the TNM classification of gastric carcinoma [5]. Accordingly, tumors were pathologically divided into four T-categories: pT1, pT2, pT3, and pT4.

Tumor size was measured according to the Japanese Classification of Gastric Carcinoma (3rd English edition) [17]. The resected specimen was opened along the greatest curvature and placed on a flat board. The maximum tumor diameter was recorded as tumor size and used for further analysis in this study. Furthermore, tumor spread was pathologically reconfirmed.

\section{Statistical analysis}

The relationship between tumor depth and categorical clinicopathological factors was assessed using the Chi-squared test or Fisher's exact test. Differences in the relationship between tumor size and clinicopathological factors were evaluated using the Wilcoxon rank-sum test. ROC curves were constructed and the AUC was calculated to assess the predictive power of tumor size to discriminate patients with lymph node metastasis from those without lymph node metastasis. The relationship between PTS and clinicopathological features was analyzed using the Chi-squared test or Fisher's exact test. Kaplan-Meier survival curves were generated and prognostic differences were determined using log-rank testing. Prognostic factors were assessed by uni- and multivariate analyses (Cox proportional hazards regression modeling). All data were analyzed using SAS statistical software (SAS Institute, Cary, NC). A value of $P<0.05$ was considered statistically significant.

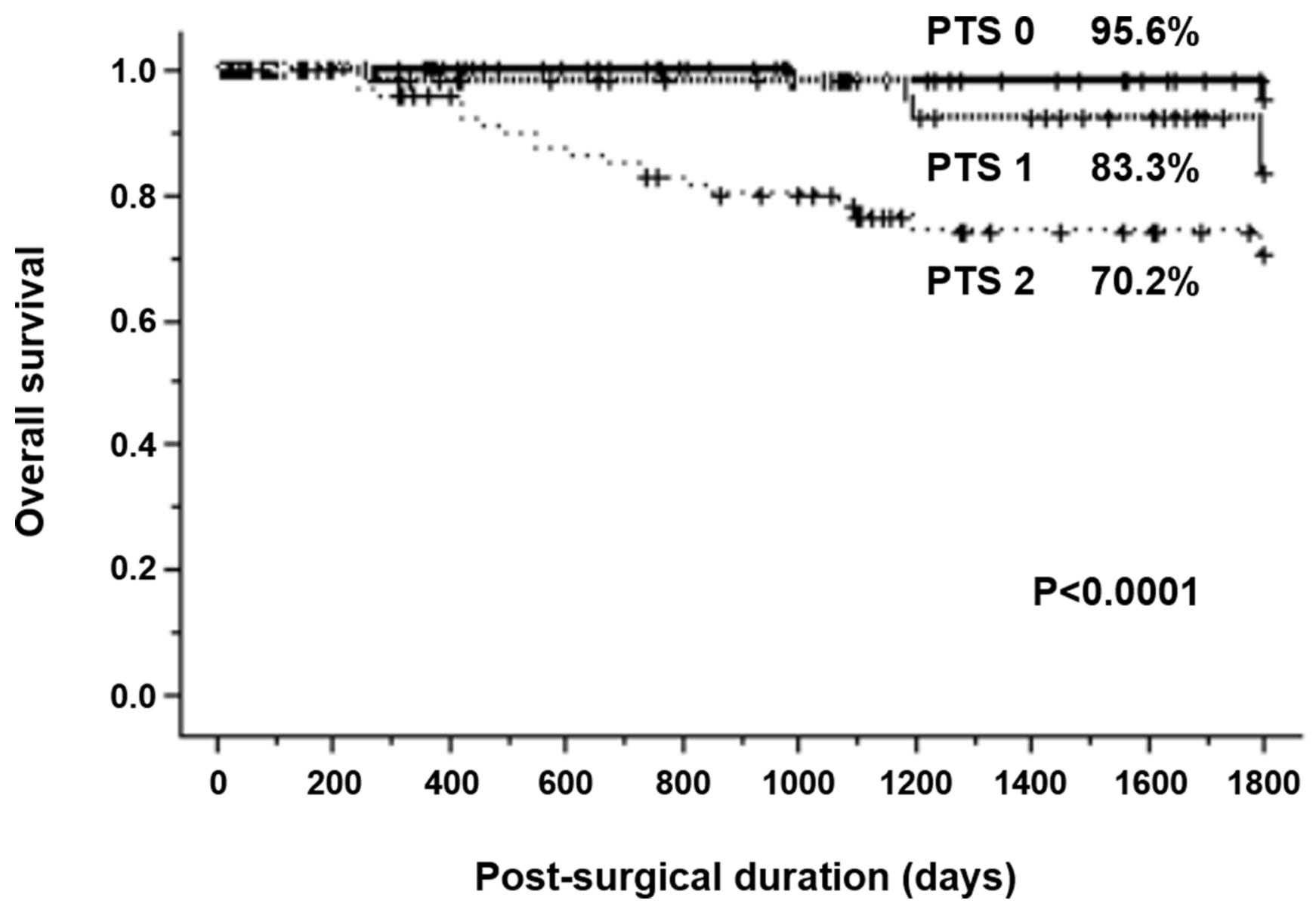

Figure 2: Kaplan-Meier survival curves for patients with resectable gastric cancer based on primary tumor score. 
Sex

Male

Female

Age (y)

$\leq 70$

137

$>70$

Tumor location

Upper

Middle

Lower

Depth of tumor invasion

pT1

pT2

pT3

pT4

Lymph node metastasis

Negative

Positive

Stage

I

II

III

Lymphatic invasion

Negative

Positive

Venous invasion

Negative

Positive

Histological type

Differentiated

Undifferentiated

Serum CEA levels $(<5 \mathrm{ng} / \mathrm{mL})$

Negative

Positive

Unknown

Serum CA 19-9 levels $(<37$ U/mL)

Negative

Positive

Unknown

pT1: invasion of lamina propria or submucosa; pT2: invasion of muscularis propria; $\mathrm{pT} 3$ : invasion of subserosa; $\mathrm{pT} 4$ : penetration of serosa or invasion of adjacent structures. 


\section{CONFLICTS OF INTEREST}

The authors have no conflicts of interest to disclose.

\section{FUNDING}

This work was supported in part by grants-in-aid (no. 16K10508) for scientific research from the Ministry of Education, Science, Sports, and Culture, Japan.

\section{REFERENCES}

1. Fitzmaurice C, Dicker D, Pain A, Hamavid H, MoradiLakeh M, MacIntyre MF, Allen C, Hansen G, Woodbrook R, Wolfe C, Hamadeh RR, Moore A; and Global Burden of Disease Cancer Collaboration. The Global Burden of Cancer 2013. JAMA Oncol. 2015; 1:505-27.

2. Japanese Gastric Cancer Association. Japanese gastric cancer treatment guidelines 2014 (ver. 4). Gastric Cancer. 2017; 20:1-19.

3. Webb A, Scott-Mackie P, Cunningham D, Norman A, Andreyev J, O'Brien M, Bensted J. The prognostic value of serum and immunohistochemical tumour markers in advanced gastric cancer. Eur J Cancer. 1996; 32:63-68.

4. Gaspar MJ, Arribas I, Coca MC, Diez-Alonso M. Prognostic value of carcinoembryonic antigen, CA 19-9 and CA 72-4 in gastric carcinoma. Tumour Biol. 2001; 22:318-322.

5. Edge SB, Byrd DR, Compton CC, Fritz AG, Greene FL, Trotti A, Editors. AJCC Cancer Staging Manual, ed 7. New York, Springer, 2010.

6. Adachi Y, Oshiro T, Mori M, Maehara Y, Sugimachi K. Tumor size as a simple prognostic indicator for gastric carcinoma. Ann Surg Oncol. 1997; 4:137-140.

7. Kunisaki C, Makino H, Takagawa R, Oshima T, Nagano Y, Kosaka T, Ono HA, Otsuka Y, Akiyama H, Ichikawa Y, Shimada H. Tumor diameter as a prognostic factor in patients with gastric cancer. Ann Surg Oncol. 2008; 15:1959-1967.

8. Wang X, Wan F, Pan J, Yu GZ, Chen Y, Wang JJ. Tumor size: a non-neglectable independent prognostic factor for gastric cancer. J Surg Oncol. 2008; 97:236-240.
9. Aoyama T, Yoshikawa T, Watanabe T, Hayashi T, Ogata T, Cho H, Tsuburaya A. Macroscopic tumor size as an independent prognostic factor for stage II/III gastric cancer patients who underwent D2 gastrectomy followed by adjuvant chemotherapy with S-1. Gastric Cancer. 2011; 14:274-278.

10. Guo P, Li Y, Zhu Z, Sun Z, Lu C, Wang Z, Xu H. Prognostic value of tumor size in gastric cancer: an analysis of 2,379 patients. Tumour Biol. 2013; 34:1027-1035.

11. Lu J, Huang CM, Zheng CH, Li P, Xie JW, Wang JB, Lin JX. Consideration of tumor size improves the accuracy of TNM predictions in patients with gastric cancer after curative gastrectomy. Surg Oncol. 2013; 22:167-171.

12. Zu H, Wang F, Ma Y, Xue Y. Stage-stratified analysis of prognostic significance of tumor size in patients with gastric cancer. PLoS One. 2013; 8:e54502.

13. Huang $\mathrm{CM}, \mathrm{Xu} \mathrm{M}$, Wang JB, Zheng $\mathrm{CH}$, Li P, Xie JW, Lin $\mathrm{JX}, \mathrm{Lu} \mathrm{J}$. Is tumor size a predictor of preoperative $\mathrm{N}$ staging in T2-T4a stage advanced gastric cancer? Surg Oncol. 2014; 23:5-10.

14. Deng J, Zhang R, Pan Y, Ding X, Cai M, Liu Y, Liu H, Bao T, Jiao X, Hao X, Liang H. Tumor size as a recommendable variable for accuracy of the prognostic prediction of gastric cancer: a retrospective analysis of 1,521 patients. Ann Surg Oncol. 2015; 22:565-572.

15. Zhao LY, Zhang WH, Chen XZ, Yang K, Chen XL, Liu K, Zhang B, Chen ZX, Chen JP, Zhou ZG, Hu JK. Prognostic Significance of Tumor Size in 2405 Patients With Gastric Cancer: A Retrospective Cohort Study. Medicine (Baltimore). 2015; 94:e2288.

16. Ohashi T, Komatsu S, Ichikawa D, Kosuga T, Okamoto K, Konishi H, Shiozaki A, Fujiwara H, Otsuji E. Tumor Index as a Combined Indicator of Tumor Depth and Size in Gastric Cancer. Anticancer Res. 2016; 36:1895-1900.

17. Japanese Gastric Cancer Association. Japanese classification of gastric carcinoma: 3rd English edition. Gastric Cancer. 2011; 14:101-112. 